\title{
Acerca del ventilador mecánico como recurso divisible ante la pandemia de COVID-19
}

\section{About the mechanical ventilator as a shared resource for the COVID-19 pandemic}

\author{
Jorge A. Castañón-González, * Luis A. Gorordo-Delsol, Jessica Garduño-López y \\ Marcos A. Amezcua-Gutiérrez \\ Secretaría de Salud, Hospital Juárez de México, Unidad de Cuidados Intensivos y Medicina Crítica, Ciudad de México, México
}

Leímos con interés el artículo "El ventilador mecánico como recurso divisible ante la pandemia de COVID-19", del doctor Gilberto Vázquez-de Anda et al., ${ }^{1}$ que consideramos de actualidad y de coyuntura en esta pandemia. En ese artículo, los autores concluyen que, conforme al principio de justicia distributiva que prevalece en los casos de pandemia, un ventilador mecánico puede ser considerado un recurso divisible y ser compartido por al menos dos pacientes en un mismo momento.

Al margen de las consideraciones fisiológicas y clínicas que ya demostraron que es factible ventilar a dos pacientes con un solo ventilador, ${ }^{2-4}$ tenemos los siguientes comentarios:

La medicina, las leyes y los valores sociales no son estáticos, por ende, reexaminar los principios éticos de la medicina y su aplicación en circunstancias nuevas o extraordinarias (como esta pandemia) es un ejercicio obligado de la sociedad, en particular de los médicos. Consideramos que las dudas no terminan aquí: ¿cuándo es divisible y cuándo no?, ¿qué otros procedimientos o equipos, antes considerados indivisibles, pueden adaptarse para compartir entre pacientes?

En nuestra profesión se depositan conocimientos, habilidades y actitudes de gran valor para la sociedad, que se expresan mediante el profesionalismo. Todo este bagaje no debe circunscribirse solo a la enfermedad del paciente en lo individual, sino extenderse a la sociedad y su circunstancia; en otras palabras, reconciliar la medicina tradicional enfocada en el paciente con la salud pública, reconociendo la verdadera dimensión del quehacer médico. Es fundamental vislumbrar escenarios futuros con este enfoque y en estos tiempos de incertidumbre o escasez de recursos. El trabajo motivo de esta carta es un ejemplo de cómo la investigación impacta a la práctica y a la ética médica en situaciones extraordinarias.

\section{Bibliografía}

1. Vázquez-de Anda G, Ruíz-de Chávez M, Pérez-Castañeda A, Vázquez-Moreno P, Dávila-Fernández JC, Delaye-Aguilar MG. El ventilador mecánico como recurso divisible ante la pandemia de COVID-19. Gac Med Mex. 2020;156:306-310.

2. Beitler JR, Mittel AM, Kallet R, Kacmarek R, Hess D, Branson R, et al. Ventilator sharing during an acute shortage caused by COVID-19 pandemic. Am J Respir Crit Care Med. 2020;202:600-604.

3. Castañón-González JA, Camacho-Juárez S, Gorordo-Delsol LA, Garduño-López J, Pérez-Nieto O, Amezcua-Gutiérrez MA, et al. Ventilación mecánica simultánea con un solo ventilador a varios pacientes. Gac Med Mex. 2020;156:250-253.

4. Castañón-González JA, Camacho-Juárez S, Gorordo-Delsol LA, Garduño-López J, Pérez-Nieto O, Amezcua-Gutiérrez MA, et al. Factibilidad de la ventilación mecánica compartida. Gac Med Mex. 2020;156:368.

\section{Correspondencia:}

*Jorge A. Castañón-González

E-mail: jorge.castanong@gmail.com

0016-3813/@ 2020 Academia Nacional de Medicina de México, A.C. Publicado por Permanyer. Este

CC BY-NC-ND (http://creativecommons.org/licenses/by-nc-nd/4.0/).
Gac Med Mex. 2020;156:486

Disponible en PubMed www.gacetamedicademexico.com 